\title{
Realization of Poincaré group induced by a second-order ordinary differential system. Noninteraction theorem
}

\author{
$X$. Jaen and J. Llosa \\ Grup de Relativitat de la Secció de Física (SCC) IEC, Departament de Física Teòrica, Universitat de \\ Barcelona, 08028 Barcelona, Spain \\ F. Marqués \\ Grup de Relativitat de la Secció de Física (SCC) IEC, Universitat Politècnica de Catalunya (ETSECCP), \\ 08028 Barcelona, Spain
}

\author{
A. Montoto \\ Grup de Relativitat de la Secció de Física (SCC) IEC, Departament de Física Teòrica, Universitat de \\ Barcelona, 08028 Barcelona, Spain
}

(Received 31 January 1985; accepted for publication 16 October 1985)

\begin{abstract}
A generalization of the predictive relativistic mechanics is studied where the initial conditions are taken on a general hypersurface of $M^{4}$. The induced realizations of the Poincare group are obtained. The same procedure is used for the Galileo group. Noninteraction theorems are derived for both groups.
\end{abstract}

\section{INTRODUCTION}

The generalization of the no-interaction theorem presented here is undertaken in the framework of predictive relativistic mechanics (PRM), ${ }^{1}$ that is, the same Newtonian equations of motion remain valid for every inertial observer. Relativistic invariance is then understood as referring to worldlines, thus adopting the standpoint first stated by Minkowski $^{2}$ that "...physical laws might find their most perfect expression as reciprocal relations between these worldlines."

In the usual formulation of PRM, the configuration space for an $N$-point particle system is spanned by the $3 N$ simultaneous position coordinates of the particles (simultaneity here presumes an inertial observer describing the dynamics of the system). In this respect, the usual PRM approach is similar to the instant form of $\mathrm{Dirac}^{3}$ for Hamiltonian relativistic dynamics.

Also, in most of the various derivations of the noninteraction theorem, the instant form assumption plays a crucial role: the simultaneous position of particles are assumed to be either canonical coordinates in the Hamiltonian approaches ${ }^{4}$ or the variables spanning the configuration space in the Lagrangian formulations. ${ }^{5}$

Other derivations of the theorem, that will not be considered here, correspond to the covariant formalism of PRM (see Ref. 6) or to the Hamiltonian relativistic systems with constraints approach. ${ }^{7}$ An interesting review on the subject can be found in Ref. 8.

Dirac proposed ${ }^{3}$ two other possible formulations of $\mathrm{Ha}$ miltonian relativistic dynamics besides the instant form: namely, the front form and the point form. So the question arose of whether the instant form assumption was essential to the noninteraction result, or if a similar output could be obtained in the framework of the other two Dirac forms. This point has been studied in a recent work ${ }^{9}$ and the answer is yes.

At this point, why should we restrict ourselves to the three Dirac forms of Hamiltonian relativistic dynamics? From a historical point of view, it becomes apparent that
Dirac proposed these three forms as different possible ways of simplification for a wider problem: the derivation of Poisson realizations for the Poincare algebra. Nevertheless, Dirac himself proposed, and later developed, a techniquerelativistic Hamiltonian dynamics with constraints ${ }^{10}$ which permits us to obtain a much wider solution to this problem, beyond the rigid restrictions of the above-mentioned three forms of dynamics.

So, as far as the noninteraction result is concerned, the following question becomes legitimate: Does it hold beyond the narrow framework of the three forms of Dirac?

A first answer to this question has been given partially ${ }^{7}$ in the framework of Hamiltonian relativistic systems with constraints. Under some assumptions it has been proved by an example that some mass-shell constraints and fixations could be chosen such that, albeit positions of particles are taken as canonical coordinates, the noninteraction implications are circumvented. However, that model has not any interest beyond the mathematical one: not only is it unphysical, but also the procedure to reconstruct the particles' worldlines from the phase space trajectories is rather sophisticated, owing to the fact that the fixations are chosen not to have a clear kinematical meaning, but to yield some wanted specific Dirac brackets.

We are going to undertake another generalization of the noninteraction theorem, now always keeping in mind the kinematic aspects of the problem, that is, the way any given inertial observer will have to recover the particles' worldlines from the configuration space trajectories. To this end, let us analyze how it is done in the instant form approach. For every given value $\lambda$ of a certain parameter, an inertial observer takes the space coordinates $x_{a}^{i}(\lambda), a=1, \ldots, N$, of each particle when

$$
x_{1}^{0}=x_{2}^{0}=\cdots=x_{N}^{0}=\lambda,
$$

and the configuration space curve $\left(x_{1}^{i}(\lambda), \ldots, x_{N}^{i}(\lambda)\right)$ describes the evolution of the system. Conversely, the $N$ worldlines are recovered from a given configuration space trajectory 
$\left(x_{1}^{i}(\lambda), \ldots, x_{N}^{i}(\lambda)\right)$, by merely taking $\left(\lambda, x_{a}^{i}(\lambda)\right), a=1, \ldots, N$.

Other requirements in PRM are (i) that the trajectories of the system are the integrals of a second-order differential system on the configuration space and (ii) the relativistic invariance in terms of worldlines. A further development of the latter two conditions yields the so-called induced realization of the Poincare group on the cophase space and the Currie-Hill equations. ${ }^{1}$

The front form (resp. point form) approach operates in a quite similar way. ${ }^{9}$ For every given value $\lambda$ of a certain parameter, an inertial observer takes the space coordinates $x_{a}^{i}(\lambda), a=1, \ldots, N$ when

$$
\begin{aligned}
& x_{a}^{0}+x_{a}^{3}=\lambda \\
& \left(\text { resp. } x_{a}^{02}-\mathbf{x}_{a}^{2}=\lambda^{2}\right) .
\end{aligned}
$$

The evolution of the system in the configuration space is then given by $\left(x_{1}^{i}(\lambda), \ldots, x_{N}^{i}(\lambda)\right)$. Conversely, for a certain configuration space curve, the worldline of the ath particle is given by

$$
\begin{aligned}
& \left(\lambda-x_{a}^{3}(\lambda), x_{a}^{i}(\lambda)\right), \quad a=1, \ldots, N, \\
& {\left[\text { resp. }\left(\sqrt{\lambda^{2}+\mathbf{x}_{a}^{2}(\lambda)}, x_{a}^{i}(\lambda)\right), a=1, \ldots, N\right] .}
\end{aligned}
$$

Similarly, the requirements of relativistic invariance and that configuration space trajectories fulfill the second-order differential system

$$
\frac{d^{2} x_{a}^{i}}{d \lambda^{2}}=a_{a}^{i}\left(\mathbf{x}_{b}, \mathbf{v}_{c}, \lambda\right)
$$

also lead to an induced realization of Poincare group on the extended cophase space and to some restrictions on the accelerations that play the same role as the Currie-Hill conditions do in the instant form approach.

In Sec. II, we shall start from an analysis of the common features of these three approaches, in order to generalize the predictive relativistic mechanics framework. Then, in Sec. III, we prove a generalization of the noninteraction theorem. Finally, in Sec. IV, we extend the previous study to Newtonian dynamics, analyzing the noninteraction theorem in this case.

\section{THE GENERALIZED PREDICTIVE RELATIVISTIC MECHANICS FRAMEWORK}

The three approaches we commented on at the end of last section (resp. instant, front, and point forms) share the following common features.

(i) Newtonian equations of motion: The configuration space of the $N$-point particle system is spanned by the $3 N$ position coordinates of the particles $x_{a}^{i}, a=1, \ldots, N$, $i=1,2,3$, and the evolution is governed by a second-order differential system

$$
\frac{d x_{a}^{i}}{d \lambda}=v_{a}^{i}, \quad \frac{d v_{a}^{i}}{d \lambda}=a_{a}^{i}\left(x_{b}^{i}, v_{c}^{k}, \lambda\right),
$$

whose functional form does not depend on the inertial observer describing the dynamics.

(ii) $A$ specific rule to construct the worldlines: For each particular solution

$$
x_{a}^{i}(\lambda)=\varphi_{a}^{i}\left(x_{b}^{j}, y_{c}^{k}, \lambda_{0} ; \lambda\right), \quad a=1, \ldots, N,
$$

of (2.1) with the initial condition

$$
\varphi_{a}^{i}\left(x, y_{b}, \lambda_{0} ; \lambda_{0}\right)=x_{a}^{i}, \quad \frac{\partial \varphi_{a}^{i}}{\partial \lambda}\left(x_{a}, y_{b}, \lambda_{0} ; \lambda_{0}\right)=y_{a}^{i},
$$

the worldline $x_{a}^{\mu}(\lambda)$ for the $a$ th particle is obtained by taking the space coordinates $(2.2)$ and finding the time coordinate $x_{a}^{0}(\lambda) \equiv \varphi_{a}^{0}\left(x_{b}^{j}, \psi_{c}^{k}, \lambda_{0} ; \lambda\right)$ from

$$
h\left(x_{a}^{\mu}(\lambda)\right)=\lambda,
$$

where $h\left(x^{\mu}\right)$ is a fixed function on space-time $M_{4}$-the same function for every inertial observer and every particle [remember Eqs. (1.1), (1.2), and (1.3) for the three forms commented on above].

Intuitively, this means that each inertial observer constructs his configuration space by taking the space coordinates of all particles when their worldlines cross a previously chosen parameterized set of space-time hypersurfaces: $h\left(x^{\mu}\right)=\lambda$.

In order that $x_{a}^{0}(\lambda)$ can be obtained from the implicit equation (2.4), the partial derivative $\partial h / \partial x^{0}$ must not vanish.

(iii) Relativistic invariance of worldlines: Let $\mathscr{S}$ and $\mathscr{S}^{\prime}$ be two inertial observers connected by the Poincaré transformation

$$
x^{\mu \mu}=L^{\mu}{ }_{v} \cdot\left(x^{\nu}-A^{\nu}\right) .
$$

Let us assume that the worldlines $\varphi_{a}^{\mu}\left(\mathbf{x}_{b}, \mathbf{v}_{c}, \lambda_{0} ; \lambda\right)$ are obtained by $\mathscr{S}$ from some initial data $\left(\mathbf{x}_{b}, \mathbf{v}_{c}, \lambda_{0}\right)$. Then, the transformed space-time curves

$$
L^{\mu}{ }_{v} \cdot\left[\varphi_{a}^{v}\left(\mathbf{x}_{b}, \mathbf{v}_{c}, \lambda_{0} ; \lambda\right)-A^{v}\right]
$$

must be such that are obtained by $\mathscr{S}^{\prime}$ starting from another set of initial data $\left(\mathbf{x}_{a}^{\prime}, \mathbf{v}_{b}^{\prime}, \lambda_{0}\right)$. That is,

$L^{\mu}{ }_{v} \cdot\left[\varphi_{a}^{v}\left(\mathbf{x}_{b}, \mathbf{v}_{c}, \lambda_{0} ; \lambda\right)-A^{\nu}\right]=\varphi_{a}^{\mu}\left(\mathbf{x}_{b}^{\prime}, \mathbf{v}_{c}^{\prime}, \lambda_{0} ; \lambda_{a}^{\prime}\right)$,

for every $\lambda$. The parameter $\lambda_{a}^{\prime}$ of the right-hand side is determined by the condition

$$
\begin{aligned}
& \lambda_{a}^{\prime}\left(\mathbf{x}_{b}, \mathbf{v}_{c}, \lambda_{0} ; L^{\mu}{ }_{\nu}, A^{\mu} ; \lambda\right) \\
& \quad=h\left(L^{\mu}{ }_{\nu} \cdot\left[\varphi_{a}^{v}\left(\mathbf{x}_{b}, \mathbf{v}_{c}, \lambda_{0} ; \lambda\right)-A^{\nu}\right]\right),
\end{aligned}
$$

which stems from requiring (2.4) to hold also for $\mathscr{S}^{\prime}$.

It can be easily obtained from (2.4) that

$$
\lambda ;\left(\mathbf{x}_{b}, \mathbf{v}_{c}, \lambda_{0} ; \delta_{v}^{\mu}, 0 ; \lambda\right)=\lambda \text {. }
$$

The new $\mathscr{S}^{\prime}$ initial data $\left(\mathbf{x}_{a}^{\prime}, \mathbf{v}_{b}^{\prime}, \lambda_{0}\right)$ will depend on the former $\mathscr{S}$ ones $\left(\mathbf{x}_{b}, \mathbf{v}_{c}, \lambda_{0}\right)$ and on the Poincaré transformation, $\left(L^{\mu}{ }_{\nu}, A^{\mu}\right) \in \mathscr{P}$, which relates $\mathscr{S}^{\prime}$ to $\mathscr{S}$. That is,

$$
\begin{aligned}
& x_{a}^{i \prime}=f_{a}^{i}\left(\mathbf{x}_{b}, \mathbf{v}_{c}, \lambda_{0} ; L^{\mu}{ }_{\nu}, A^{\mu}\right), \\
& v_{a}^{i \prime}=g_{a}^{i}\left(\mathbf{x}_{b}, \mathbf{v}_{c}, \lambda_{0} ; L^{\mu}{ }_{v}, A^{\mu}\right) .
\end{aligned}
$$

We are not going to derive explicit expressions for these functions, $f_{a}^{i}$ and $g_{b}^{j}$, which define the Poincare transformation induced by the given $\left(L^{\mu}{ }_{v}, A^{\mu}\right) \in \mathscr{P}$ on the extended cophase space $\Gamma(6 N+1)$, nor are we going to prove by a direct manipulation that they form an actual group realization. Instead, a close examination of commutation relations will ultimately prove this point. ${ }^{11}$

Note that induced Poincaré transformations act as

$$
\left(\mathbf{x}_{a}, \mathbf{v}_{b}, \lambda_{0}\right) \rightarrow\left(\mathbf{x}_{a}^{\prime}, \mathbf{v}_{b}^{\prime}, \lambda_{0}\right)
$$

thus leaving invariant, by prescription, the sheets $\lambda=$ const, 
of $\Gamma(6 N+1)$. In any parametrization $\left(L^{\mu}{ }_{\nu}\left(\epsilon_{I}\right), A^{\mu}\left(\epsilon_{J}\right)\right)$, $I, J=1, \ldots, 10$, the infinitesimal generators for induced Poincaré transformations are given by

$$
\mathbf{\Lambda}_{I}^{*}=\sum_{a}\left[\left(\frac{\partial f_{a}^{i}}{\partial \epsilon_{I}}\right)_{(\epsilon)=0} \cdot \frac{\partial}{\partial x_{a}^{i}}+\left(\frac{\partial g_{a}^{i}}{\partial \epsilon_{I}}\right)_{(\epsilon)=0} \cdot \frac{\partial}{\partial v_{a}^{i}}\right]
$$

[summation over repeated space (Roman) or space-time (Greek) indices will be hereafter understood].

The coefficients on the right-hand side of (2.11) can be obtained by taking partial derivatives with respect to $\epsilon_{I}$ and then making $\left(\epsilon_{J}\right)=(0)$ in the expression

$$
\begin{aligned}
& \varphi_{a}^{\mu}\left(f_{b}^{i}\left(\mathbf{x}, \mathbf{v}, \lambda_{0} ; \epsilon_{I}\right), g_{c}^{j}\left(\mathbf{x}, \mathbf{v}, \lambda_{0} ; \epsilon_{I}\right), \lambda_{0} ;\right. \\
&\left.h\left(L^{\rho}{ }_{\sigma} \cdot\left[\varphi_{a}^{\sigma}\left(\mathbf{x}, \mathbf{v}, \lambda_{0} ; \lambda\right)-A^{\sigma}\right]\right)\right) \\
&=L^{\mu}{ }_{\nu} \cdot\left[\varphi_{a}^{v}\left(\mathbf{x}_{b}, \mathbf{v}_{c}, \lambda_{0} ; \lambda\right)-A^{\nu}\right],
\end{aligned}
$$

which results from considering (2.7), (2.8), and (2.10) together.

Since Eq. (2.12) holds for every value of $\lambda$-at least in an open neighborhood-it can be proved easily that any induced Poincaré transformation commutes with dynamical evolution. That is, the diagram

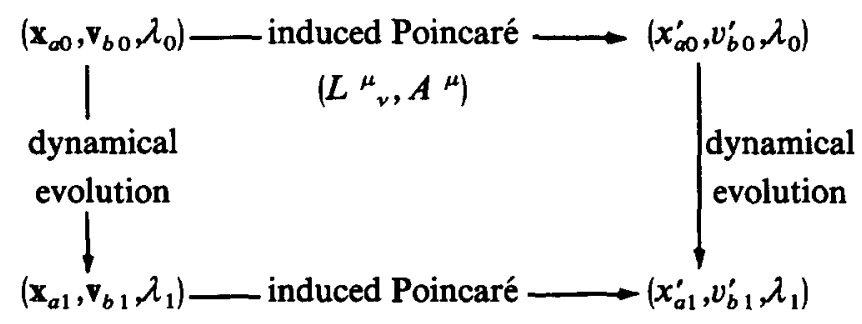

$$
\left(L^{\mu}{ }_{v}, A^{\mu}\right)
$$

is commutative.

In terms of infinitesimal generators, this condition is equivalent ${ }^{11}$ to the vanishing of the Lie brackets

$$
\left[\mathbf{\Lambda}_{i}^{*}, \mathbf{D}\right]=0 \text {, }
$$

where

$\mathbf{D}=\sum_{a}\left[v_{a}^{i} \cdot \frac{\partial}{\partial x_{a}^{i}}+a_{a}^{i}\left(\mathbf{x}, \mathbf{v}, \lambda_{0}\right) \cdot \frac{\partial}{\partial v_{a}^{j}}\right]+\frac{\partial}{\partial \lambda_{0}}$

is the infinitesimal generator of dynamical evolution on $\Gamma(6 N+1)$.

In order to find out the coefficients of the generators $\Lambda_{I}^{*}$, we infer from (2.12) that

$\Lambda_{I}^{*} x_{a}^{i}=C_{I 0}^{i} \cdot \varphi_{a}^{0}+C_{I j}^{i} \cdot x_{a}^{j}-C_{I}^{i}-v_{a}^{i} \cdot\left(\Lambda_{I} h\right)\left(\mathbf{x}_{a}, \varphi_{a}^{0}\right)$,

where

$$
C_{I v}^{\mu} \equiv\left(\frac{\partial L_{v}^{\mu}}{\partial \epsilon_{I}}\right)_{(\epsilon)=0}, \quad C_{I}^{\mu} \equiv\left(\frac{\partial A^{\mu}}{\partial \epsilon_{i}}\right)_{(\epsilon)=0},
$$

$\varphi_{a}^{0} \equiv \varphi_{a}^{0}\left(\mathbf{x}_{a}, \lambda_{0}\right)$ is the solution of

$$
h\left(\mathbf{x}_{a}, \varphi_{a}^{0}\right)=\lambda_{0}
$$

and the meaning of $\left(\boldsymbol{\Lambda}_{I} h\right)$ is explained in detail in the Appendix.

Second, from (2.13) and (2.14) we have that

$$
\mathbf{\Lambda}_{I}^{*} v_{a}^{i}=\mathbf{\Lambda}_{I}^{*}\left(\mathbf{D} x_{a}^{i}\right)=\mathbf{D}\left(\mathbf{\Lambda}_{I}^{*} x_{a}^{i}\right) \text {, }
$$

and therefore

$$
\begin{aligned}
\boldsymbol{\Lambda}_{I}^{*} v_{a}^{i}= & C_{I 0}^{i} \cdot\left(\mathbf{D} \varphi_{a}^{0}\right)+C_{I j}^{i} \cdot v_{a}^{j}-v_{a}^{i} \cdot \mathbf{D}\left(\left(\boldsymbol{\Lambda}_{I} h\right)\left(\mathbf{x}_{a}, v_{a}^{0}\right)\right) \\
& -a_{a}^{i}\left(\mathbf{x}_{b}, \mathbf{v}_{c}, \lambda_{0}\right) \cdot\left(\boldsymbol{\Lambda}_{I} h\right)\left(\mathbf{x}_{a}, \varphi_{a}^{0}\right) .
\end{aligned}
$$

Finally, by applying the commutator (2.13) to $v_{a}^{i}$, we obtain

$$
\begin{aligned}
\mathbf{\Lambda}_{I}^{*} a_{a}^{i}= & \mathbf{D} \boldsymbol{\Lambda}_{I}^{*} v_{a}^{i} \\
= & C_{I 0}^{i} \cdot \mathbf{D}^{2} \varphi_{a}^{0}+C_{I j}^{i} \cdot \mathbf{a}_{a}^{j}-\left(\mathbf{D} a_{a}^{i}\right) \cdot\left(\mathbf{\Lambda}_{I} h\right) \\
& -2 \mathbf{D}\left(\boldsymbol{\Lambda}_{I} h\right) \cdot a_{a}^{i}-v_{a}^{i} \cdot \mathbf{D}^{2}\left(\boldsymbol{\Lambda}_{I} h\right),
\end{aligned}
$$

which must be understood as necessary conditions on the accelerations $a_{a}^{i}\left(\mathbf{x}_{b}, \mathbf{v}_{c}, \lambda\right)$ for the relativistic worldline invariance (2.7) to be accomplished. These conditions will play the same role as Currie-Hill equations ${ }^{1}$ in the instant form of PRM.

At this point, we can prove that the commutation relations among the generators $\Lambda_{I}^{*}, I=1, \ldots, 10$, are precisely those of the Poincare group. First, after a simple but rather tedious calculation, we arrive at

$$
\left[\Lambda_{I}^{*}, \Lambda_{J}^{*}\right] x_{a}^{i}=C_{I J}^{K} \cdot \Lambda_{K}^{*} x_{a}^{i} .
$$

Second, taking (2.13) and (2.20) into account, we have

$$
\left[\mathbf{\Lambda}_{I}^{*}, \mathbf{\Lambda}_{J}^{*}\right] v_{a}^{i}=C_{I J}^{K} \cdot \mathbf{\Lambda}_{K}^{*} v_{a}^{i},
$$

and, since $\Lambda_{l}^{*} \lambda_{0}=0$, we finally obtain

$$
\left[\Lambda_{I}^{*}, \Lambda_{J}^{*}\right]=C_{I J}^{K} \cdot \Lambda_{K}^{*} \text {, }
$$

where $C_{I J}^{K}, I, J, K=1, \ldots, 10$, stand for the structure constants of the Lie algebra of Poincaré. (See the Appendix.)

\section{NONINTERACTION THEOREM}

Let us now assume that there is a Poisson bracket structure $^{12}$ of maximum rank on $\Gamma(6 N+1)$ such that we have the following.

(i) The coordinate $\lambda_{0}$ of $\Gamma(6 N+1)$ is neutral relative to this Poisson bracket, i.e.,

$$
\left\{\lambda_{0}, f\right\}=0,
$$

for every function on $\Gamma(6 N+1)$.

(ii) Induced Poincaré transformations are canonical. That is, there exist ten generating functions $\Lambda_{I}^{*}\left(\mathbf{x}, \mathbf{v}, \lambda_{0}\right)$, $I=1, \ldots, 10$, such that

$$
\Lambda_{I}^{*} f=\left\{\Lambda_{I}^{*}, f\right\},
$$

for every function $f$.

(iii) The $3 N$ position coordinates $x_{a}^{i}$ can be complemented with $3 N$ conjugated momenta $p_{j}^{b}\left(\mathbf{x}, \mathbf{v}, \lambda_{0}\right)$ thereby obtaining a set of canonical variables whose elementary Poisson brackets are

$$
\begin{aligned}
& \left\{x_{a}^{i}, x_{b}^{j}\right\}=0, \\
& \left\{x_{a}^{i}, p_{j}^{b}\right\}=\delta_{a}^{b} \cdot \delta_{j}^{i}, \quad\left\{p_{i}^{b}, p_{j}^{a}\right\}=0 .
\end{aligned}
$$

As is well known - the proof can be found in any treatise on advanced analytical mechanics ${ }^{13}$-Eq. (3.2) is the necessary and sufficient condition for the differential system (3.3) to have a solution.

Substituting $f$ in Eq. (3.1) by either $x_{a}^{j}$ or $v_{b}^{i}$, and taking (2.14) $-(2.16)$ into account, we have

$$
\left\{\Lambda_{I}^{*}, x_{a}^{i}\right\}=C_{I 0}^{i} \cdot \varphi_{a}^{0}+C_{l j}^{i} x_{a}^{j}-C_{I}^{i}-v_{a}^{i} \cdot\left(\Lambda_{I} h\right)\left(\mathbf{x}_{a}, \varphi_{a}^{0}\right),
$$




$$
\begin{aligned}
\left\{\Lambda_{I}^{*}, v_{a}^{i}\right\}= & C_{I 0}^{i} \cdot\left(\mathbf{D} \varphi_{a}^{0}\right)+C_{I j}^{i} \cdot v_{a}^{j}-a_{a}^{i} \cdot\left(\boldsymbol{\Lambda}_{I} h\right)\left(\mathbf{x}_{a}, \varphi_{a}^{0}\right) \\
& -v_{a}^{i} \cdot \mathbf{D}\left(\left(\mathbf{\Lambda}_{I} h\right)\left(\mathbf{x}_{a}, \varphi_{a}^{0}\right)\right) .
\end{aligned}
$$

Since $\lambda_{0}$ is a neutral function relatively to the Poisson bracket, taking (3.2) into account and using a known property of Poisson brackets, we can write

$$
\begin{aligned}
& \left\{x_{a}^{i}, a_{b}^{j}\right\}=\left\{x_{a}^{i}, v_{c}^{k}\right\} \frac{\partial a_{b}^{j}}{\partial v_{c}^{k}}, \\
& \left\{v_{a}^{i}, a_{b}^{j}\right\}=\left\{v_{a}^{i}, x_{c}^{k}\right\} \frac{\partial a_{b}^{j}}{\partial x_{c}^{k}}+\left\{v_{a}^{i}, v_{c}^{k}\right\} \frac{\partial a_{b}^{j}}{\partial v_{c}^{k}} .
\end{aligned}
$$

Then, by applying the Jacobi identity and taking (3.2) into account, we obtain

$$
\left\{x_{a}^{i},\left\{\Lambda_{I}^{*}, x_{b}^{j}\right\}\right\}+\left\{x_{b}^{j},\left\{x_{a}^{i}, \Lambda_{I}^{*}\right\}\right\}=0,
$$

which, using (3.4), yields

$$
\left[\left(\mathbf{\Lambda}_{I} h\right)\left(\mathbf{x}_{a}, \varphi_{a}^{0}\right)-\left(\boldsymbol{\Lambda}_{I} h\right)\left(\mathbf{x}_{b}, \varphi_{b}^{0}\right)\right] \cdot\left\{x_{b}^{j}, v_{a}^{i}\right\}=0 .
$$

This finally implies

$$
\left\{x_{b}^{j}, v_{a}^{i}\right\}=0, \quad \forall a \neq b .
$$

By repeating the same treatment with (3.9) instead of (3.2), we obtain

$$
\left\{x_{a}^{i}, a_{b}^{j}\right\}+\left\{v_{a}^{i}, v_{b}^{j}\right\} \cdot\left(\Lambda_{I} h\right)\left(\mathbf{x}_{a}, \varphi_{a}^{0}\right)=0 .
$$

Then, by considering together this expression and the one that results from interchanging the indices $b$ and $a$, and by the same reasoning, that permitted us to pass from $(3.8)$ to (3.9), we can write

$$
\left\{x_{a}^{i}, a_{b}^{j}\right\}=\left\{v_{a}^{i}, v_{b}^{i}\right\}=0, \quad \forall a \neq b,
$$

unless $\left(\boldsymbol{\Lambda}_{I} h\right)\left(\mathbf{x}_{a}, \varphi_{a}^{0}\right)=\left(\boldsymbol{\Lambda}_{I} h\right)\left(\mathbf{x}_{b}, \varphi_{b}^{0}\right)$, for every $I=1, \ldots, 10$.

Again, by repeating exactly the same procedure to $\mathbf{E q}$. (3.10), we obtain

$$
\left\{v_{a}^{i}, a_{b}^{j}\right\}=0, \quad \forall a \neq b .
$$

Then, by substituting (3.9)-(3.11) into (3.6) and (3.7) and taking into account the fact that $\left(x_{a}^{i}, v_{b}^{j}, \lambda_{0}\right)$ is a complete set of independent variables $\left(\lambda_{0}\right.$ being a neutral function), after some manipulation we have that

$$
\frac{\partial a_{b}^{j}}{\partial x_{a}^{i}}=0, \quad \frac{\partial a_{b}^{j}}{\partial v_{a}^{i}}=0, \quad \forall a \neq b .
$$

Therefore, the acceleration of a particle can only depend on the variables of the particle itself,

$$
\mathbf{a}_{b}=\mathbf{a}_{b}\left(\mathbf{x}_{b}, \mathbf{v}_{b}, \lambda_{0}\right), \quad b=1, \ldots, N,
$$

and, consequently, there is no interaction between particles. Moreover, these accelerations must be required to satisfy Eq. (2.19) —analogous to the Currie-Hill equation - which will imply further restrictions on them. Since the function $h\left(x^{\mu}\right)$ is unspecified, it is rather cumbersome to analyze in detail what these restrictions are like. However, once $h\left(x^{\mu}\right)$ is made explicit, the analysis is easier in the well-known three forms of Dirac-instant form ${ }^{4}\left(x^{0}=\lambda\right)$, front form ${ }^{9}$ $\left(x_{0}+x^{3}=\lambda\right)$, or point form ${ }^{9}\left(\lambda^{2}=-x^{\mu} x_{\mu}\right)$. Introducing (3.12) into (2.19), we obtain that accelerations must be parallel to velocity, that is, motions of particles are rectilinear and uniform.

The clue of what has been proved hitherto lies in the fact that for some generator of Poincare group we have

$$
\left(\boldsymbol{\Lambda}_{I} h\right)\left(\mathbf{x}_{a}, \varphi_{a}^{0}\right) \neq\left(\boldsymbol{\Lambda}_{I} h\right)\left(\mathbf{x}_{b}, \varphi_{a}^{0}\right), \quad a \neq b .
$$

Indeed, let us assume that there is a function $h\left(x^{\mu}\right)$ such that

$$
\begin{aligned}
& \left(\mathbf{\Lambda}_{I} h\right)\left(\mathbf{x}_{a}, \varphi_{a}^{0}\left(\mathbf{x}_{a}, \lambda_{0}\right)\right)=\left(\mathbf{\Lambda}_{I} h\right)\left(\mathbf{x}_{b}, \varphi_{b}^{0}\left(\mathbf{x}_{b}, \lambda_{0}\right)\right), \\
& \forall I=1, \ldots, 10,
\end{aligned}
$$

or, according to Eq. (A2), that

$$
\begin{aligned}
& h_{\mu}\left(\mathrm{x}_{a}, \varphi_{a}^{0}\right) \cdot\left[C_{I j}^{\mu} x_{a}^{j}+C_{I 0}^{\mu} \cdot \varphi_{a}^{0}-C_{I}^{\mu}\right] \\
& \quad=h_{\mu}\left(\mathrm{x}_{b}, \varphi_{b}^{0}\right) \cdot\left[C_{i j}^{\mu} \cdot x_{b}^{j}+C_{I 0}^{\mu} \cdot \varphi_{b}^{0}-C_{I}^{\mu}\right],
\end{aligned}
$$

which, in the case of translations, yields

$$
h_{\mu}\left(\mathbf{x}_{a}, \varphi_{a}^{0}\right)=h_{\mu}\left(\mathbf{x}_{b}, \varphi_{b}^{0}\right) \text {, }
$$

the most general solution of which is

$$
h\left(x^{\mu}\right)=a_{v} \cdot x^{\nu}+b .
$$

The requirement that (3.44) is to be fulfilled in case of rotations, $I=(i j)$, restricts a little bit more the form of $h(x)$, specifically

$$
h\left(x^{\mu}\right)=a_{0} x^{0}+b .
$$

Finally, Eq. (31.4) specialized for boosts leads to

$$
a_{0} \cdot x_{a}^{i}=a_{0} \cdot x_{b}^{i}, \quad a \neq b,
$$

which implies that $a_{0}=0$.

We have arrive at $h\left(x^{\mu}\right)=b$, constant, which contradicts the previous assumption that

$$
\frac{\partial h}{\partial x^{0}}\left(x^{\mu}\right) \neq 0 \text {. }
$$

Consequently, we have proved that is not possible in any way to choose a hypersurface-defining function $h\left(x^{\mu}\right)$ such that the noninteraction result can be avoided, provided that a canonical realization of the Poincare algebra and the canonical character of position coordinates are simultaneously assumed.

\section{A "NONINTERACTION THEOREM" IN GALILEAN DYNAMICS}

The three forms of Dirac for relativistic dynamics coincide when the Galilean limit $(c \rightarrow \infty)$ is taken, thus leading to the natural form used by physicists for centuries. In spite of this, we shall devote this section to the seemingly academic task of extending the results formally obtained to Galilean dynamics. The outcome will be pleasantly surprising.

Since the results obtained in Secs. II and III have been derived in a rather generic way, similar results will hold for any transformation group of space-time, e.g., the Galilei group.

So, we can also speak of induced Galilean transformations on the extended cophase space and all that has been presented in Sec. II holds by changing "Poincaré" to "Galileo"-this change affects the coefficients $C_{\nu}^{\mu}$ and $C_{I}^{\mu}$ and the structure constants $C_{I J}^{K}$.

Also, as has been remarked at the end of Sec. III, in order to avoid the no-interaction result, we must find a function $h(\mathbf{x}, t)$ on space-time fulfilling Eq. (3.14).

As we did in the last section, specializing (3.14) to space rotations and space-time translations, we obtain that $h$ must be

$h(\mathbf{x}, t)=a \cdot t+b$, 
if (3.14) is to be fulfilled.

And, since the generators of Galilean boosts in spacetime are $-t\left(\partial / \partial x^{i}\right),(3.14)$ is identically satisfied in this case.

We have therefore arrived at the interesting result that, even in Galilean dynamics, we could wind up with a noninteraction result if we had not chosen the suitable form-i.e., the function $h(\mathbf{x}, t)$. However, contrary to the relativistic case, Galilean dynamics permits only one way-instant form-to escape from noninteraction, and this is precisely the one that has been naively used from the beginning of Hamiltonian classical mechanics.

\section{CONCLUDING REMARKS}

The starting point of the present paper has been that the equations of motion for an $N$-point particle system are second-order differential equations and that the configuration space of each inertial observer is constructed by taking particle positions when their worldlines cross a given space-time hypersurface $h\left(x^{\mu}\right)=$ const. Relativistic invariance then has been imposed by requiring the equations of motion and the space-time hypersurface to have the same functional form for every inertial observer.

The outcome is a generalization of predictive relativistic mechanics, ${ }^{1}$ which is recovered as a particular case of the framework here developed, by taking $h\left(x^{\mu}\right)=x_{0}$. And in this generalized framework we have obtained the conditions that accelerations must fulfill if worldline relativistic invariance is required. These conditions appear as the counterpart of the well-known Currie-Hill equations of predictive relativistic mechanics.

We have proved then that the requirement of having a canonical formulation for induced Poincare transformations, where position coordinates can be taken as canonical ones, unavoidably implies noninteraction.

As a consequence of the general manner as the problem has been dealt with; the results obtained in the first part of the paper for the Poincare group can be easily translated to the case of any other group of space-time transformations. We thus have written them for the case of the Galilei group, thus concluding, in Newtonian dynamics, the only way of avoiding noninteraction theorems in the usual "instant form," which always has been used as the natural one in classical Hamiltonian mechanics.

\section{APPENDIX}

For a given function $h\left(x^{\mu}\right)$ on the Minkowski space and for the standard realization of Poincaré group, we have

$$
\left(\Lambda_{I} h\right)(x) \equiv\left(\partial_{\mu} h\right)(x)\left[C_{\nu \nu}^{\mu} x^{\nu}-C_{I}^{\mu}\right] .
$$

According to this, we define the shortened notation

$$
\begin{aligned}
\left(\boldsymbol{\Lambda}_{I} h\right)_{a} & \equiv\left(\Lambda_{I} h\right)\left(\mathbf{x}_{a}, \varphi_{a}^{0}\right) \\
& \equiv\left(\partial_{\mu} h\right)\left(\mathbf{x}_{a}, \varphi_{a}^{0}\right)\left[C_{l j}^{\mu} x_{a}^{j}+C_{I 0}^{\mu} \varphi_{a}^{0}-C_{I}^{\mu}\right],
\end{aligned}
$$

where $\varphi_{a}^{0}\left(\mathbf{x}_{a}, \lambda_{0}\right)$ is obtained by solving $(2.17)$.

On the other hand, in the usual parametrization of the Poincaré group

$$
\begin{aligned}
& L^{\mu}{ }_{\nu}=\delta^{\mu}{ }_{\nu}+\omega^{\alpha \beta} \delta_{\alpha}^{\mu} \eta_{\nu \beta}+O\left(\omega^{2}\right), \omega^{\alpha \beta}=-\omega^{\beta \alpha}, \\
& A^{\mu}=\epsilon^{\rho} \delta_{\rho}^{\mu}, \\
& A^{\mu}=\epsilon^{\rho} \delta_{\rho}^{\mu},
\end{aligned}
$$

we have that

$$
\begin{aligned}
& C_{(\alpha \beta) v}^{\mu}=\delta_{\alpha}^{\mu} \eta_{v \beta}-\delta_{\beta}^{\mu} \eta_{v \alpha}, \quad C_{(\rho) v}^{\mu}=0, \\
& C_{(\alpha \beta)}^{\mu}=0, \quad C_{(\rho)}^{\mu}=\delta_{\rho}^{\mu} .
\end{aligned}
$$

'D. G. Currie, Phys. Rev. 142, 817 (1966); R. N. Hill, J. Math. Phys. 8, 201 (1967); L. Bel, Ann. Inst. H. Poincaré 12, 307 (1970).

${ }^{2}$ H. Minkowski, "Space and time," in The Principle of Relativity (Dover, New York, 1923).

${ }^{3}$ P. A. M. Dirac, Rev. Mod. Phys. 21, 392 (1949).

${ }^{4}$ D. G. Currie, T. F. Jordan, and E. C. G. Sudarshan, Rev. Mod. Phys. 35, 350 (1963); H. Leutwyler, Nuovo Cimento 37, 556 (1965); R. N. Hill, J. Math. Phys. 8, 1756 (1967).

${ }^{5}$ E. H. Kerner, J. Math. Phys. 9, 222 (1968); J. Martin and J. L. Sanz, J. Math. Phys. 19, 780 (1978); J. Martin and J. L. Sanz, J. Math. Phys. 20, 25 (1979).

${ }^{6}$ P. Droz-Vincent, Nuovo Cimento 128, 1 (1972); L. Bel (private communication).

${ }^{7}$ A. Komar, Phys. Rev. D 18, 3617 ( 1978); V. Iranzo, J. Llosa, F. Marques, A. Molina, and A. Montoto, Phys. Rev. D 31, 314 (1985).

${ }^{8}$ S. Chelkowski, J. Nietendel, and R. Suchanek, Acta Phys. Pol. B 11, 809 (1980).

'X. Jaen, A. Molina, and V. Iranzo, "Front form and point form fomulation of predictive relativistic mechanics. Non interaction theorems," preprint (UB-FT-12.84).

${ }^{10} \mathrm{P}$. A. M. Dirac, Lectures on Quantum Mechanics (Belfer Graduate School of Science, New York, 1964).

"L. S. Pontrjagin, Grupos Contínuos (MIR, Moscow, 1978).

${ }^{12}$ E. C. G. Sudarshan and N. Mukunda, Classical Dynamics: A Modern Perspective (Wiley, New York, 1974), Chap. 9.

${ }^{13}$ L. P. Eisenhart, Continuous Groups of Transformations (Dover, New York, 1961), Chap. VI. 